\title{
Atenção à mulher no pré-natal: Análise da assistência versus direito à saúde
}

\section{Attention to women in pre-christmas: Analysis of care versus right to health}

\author{
Rosângela Nunes Almeida ${ }^{1 *}$, Francidalma Soares Sousa Carvalho Filha ${ }^{2}$, Alison de Sousa \\ Moreira ${ }^{3}$, Maria Lindalva Alves da Silva $\bullet$, Elzimar Palhano dos Santos ${ }^{5}$, Livia Cristina \\ da Silva Paiva 6, Lawanda Kelly Matias de Macedo 7
}

${ }^{1}$ Enfermeira, Mestre em Biodiversidade, Ambiente e Saúde, Universidade Estadual do Maranhão (UEMA). Caxias-MA Brasil. E-mail: rnadasilva@hotmail.com * Autor para correspondência

${ }^{2}$ Enfermeira, Doutora em Saúde Pública, Centro Universitário de Ciências e Tecnologia do Maranhão e Universidade Estadual do Maranhão (UEMA). Caxias-MA Brasil. E-mail: francidalmafilha@gmail.com

${ }^{3}$ Enfermeiro, Especialista em Urgência e Emergência, Coordenador do Serviço de Atendimento Móvel de Urgência. Aldeias Altas-MA Brasil. Email: alisonsousa2012@bol.com.br

${ }^{4}$ Bióloga, Mestre em Biodiversidade, Ambiente e Saúde, Universidade Estadual do Maranhão (UEMA). Caxias-MA Brasil. E-mail: lindalva.maria@hotmail.com

${ }^{5}$ Enfermeira. Mestre em Saúde Pública, Coordenadora do Controle, Monitoramento e Avaliação. Lago da Pedra e Alto Alegre do Pindaré-MA Brasil E-mail: elzimarpalhano@hotmail.com

${ }^{6}$ Enfermeira, Especialista em Unidade de Terapia Intensiva, Docente do Curso de Enfermagem. Anhanguera. Caxias-MA Brasil E-mail: enf.liviapaiva@hotmail.com

${ }^{7}$ Acadêmica de Enfermagem, Universidade Estadual do Maranhão (UEMA). Caxias-MA Brasil. E-mail: lawandak360@gmail.com

\section{Palavras-chave}

Atenção

Pré-Natal

Direito à Saúde
Objetivou-se analisar a assistência ofertada às mulheres no pré-natal na Atenção Primária à Saúde, na interface com o direito à saúde. Trata-se de uma pesquisa avaliativa, descritiva e exploratória, com abordagem qualitativa. O cenário da investigação foi o município de Aldeias Altas (MA), tendo sido realizado com 10 gestantes acompanhas pela Equipe de Saúde da Família 03, inserida na Unidade Básica de Saúde Centro de Saúde. Os dados foram submetidos à Análise de Conteúdo, proposta por Bardin. Os resultados revelaram que, os profissionais de saúde, realizam as orientações às gestantes durante as consultas de pré-natal, evidenciado pela satisfação das gestantes acerca dos serviços recebidos pelos profissionais da equipe. Identificou-se que quanto aos aspectos da Política Nacional de Humanização, os profissionais não seguem os preceitos da PNH, uma vez que descumpre um dos seus requisitos básicos, que é a visita domiciliar. Percebeu-se que a prática de educação em saúde está sendo realizada. Dessa forma, enfatiza-se a realização de uma assistência às gestantes com qualidade, com vistas a reduzir a ocorrência de alterações, que venham a acometer a mulher e o concepto. Ademais, ressalta-se o direito à saúde, como uma prerrogativa indispensável à apropriação da liberdade e da igualdade e a efetivação dos deveres do estado frente aos cidadãos, particularmente as mulheres que estão vivendo esse momento ímpar, que é a gravidez.

\section{Keywords}

Attention

Prenatal

Right to health
The objective was to analysis the assistance offered to women in prenatal care in Primary Health Care, in the interface with the right to health. This is an evaluative, descriptive and exploratory research with a qualitative approach. The research scenario was the municipality of Aldeias Altas (MA), and it was carried out with 10 pregnant women accompanied by the Family Health Team 03, inserted in the Basic Health Unit Health Center. The data were submitted to Content Analysis, proposed by Bardin. The results revealed that, the health professionals, provide guidelines to pregnant women during prenatal consultations, evidenced by the satisfaction of pregnant women about the services received by the professionals of the team. It was identified that as regards aspects of the National Humanization Policy, professionals do not follow the precepts of the HNP, since it fails one of its basic requirements, which is the home visit. It was noticed that the practice of health education is being carried out. Thus, it is emphasized the provision of assistance to pregnant women with quality, with a view to reducing the occurrence of changes that may affect women and the concept. In addition, the right to health is emphasized as a prerogative indispensable to the appropriation of freedom and equality and the fulfillment of the duties of the state vis-à-vis the citizens, particularly the women who are experiencing this unique moment, which is pregnancy.

\section{INTRODUÇÃO}

A Atenção à Saúde da mulher no período gestacional implica em uma assistência de qualidade e humanizada, com vistas a compreender o processo saúde-doença sob uma nova percepção. Dessa forma, é necessário entender a paciente na 
sua integralidade, singularidade e multidimensionalidade, considerando o ambiente em que ela está inserida e valorizando as suas diferenças, identidades e peculiaridades do gênero (ZAMPIERI, 2006).

De acordo com a Política Nacional de Atenção Básica, Portaria MS/GM no 2.488 de 21 de outubro de 2011 (BRASIL, 2011), a Saúde da Mulher constitui-se em uma das áreas estratégicas nacionais para atuação no âmbito da Atenção Básica em Saúde, e preconiza uma assistência em todos os níveis de saúde a esta clientela.

O acesso aos serviços de saúde é essencial para realização do cuidado pré-natal e influencia na adesão da gestante ao programa, bem como na sua qualidade, o mesmo deve ser iniciado precocemente, propiciando cobertura universal, ressaltando que deve ser realizado de forma periódica. A captação precoce das gestantes na assistência pré-natal é fator preponderante para identificação de risco nas gestações, que contribuirá a realização de intervenções mais rápidas e efetivas e a redução da morbidade e mortalidade materna e infantil (CORREA; BONADIO; TSUNECHIRO, 2011).

Nessa perspectiva, em 1984 o Ministério da Saúde implantou o Programa de Assistência Integral à Saúde da Mulher (PAISM), como estratégia para aperfeiçoar a atenção à saúde reprodutiva da mulher, por meio da qualificação dos serviços de saúde para atender as necessidades exclusivas desse público, com abordagem multiprofissional do cuidado e integralidade da atenção, utilizando um enfoque no contexto social, familiar, emocional e de prevenção de doenças e agravos (ANDREUCCI; CECATTI, 2011).

Com a implantação do Programa Saúde da Família, em 1994, atualmente denominado Estratégia Saúde da Família (ESF), a atenção à saúde da mulher, sobretudo no período gravídico, passou a ser implementada por equipes multiprofissionais compostas por médico, enfermeiro e agentes comunitários de saúde, além de outros, como os profissionais pertencentes ao Núcleo de Apoio ao Saúde da Família, a exemplo fisioterapeutas, psicólogos, nutricionistas, dentre outros. Assim, a assistência ofertada à esta clientela ganha na Atenção Primária à Saúde (APS) sua mais importante aliada na redução da morbimortalidade materna, fetal e neonatal (BRASIL, 2011).

Ressalta-se ainda que, com vistas a ampliar o leque de ações de saúde destinadas à população feminina, 01 de junho de 2000 desenvolveu-se o Programa de Humanização do PréNatal e Nascimento (PHPN) objetivando, firmar o atendimento humanizado na atenção integral e inserir artifícios para melhorar as condições de assistência à gestante na rede pública de saúde (POLIDO, 2010).

Quanto o direito à saúde, constitui-se um dos direitos fundamentais, e está expressamente protegido no caput do Art. 5o da Constituição Federal, e ainda garantido pela Lei
Orgânica da Saúde/LOS 8080/1990. A este respeito Rocha (2011), ressalta que por direito à saúde entende-se uma questão abrangente e diversa de reclame de bens e direitos nas esferas do governo, e ainda, que se referem aos insumos, instalações, medicamentos, assistência em saúde, entre outros fatores a serem protegidos pelo princípio do direito à saúde.

Neste contexto, discute-se a importância da Atenção à no pré-natal, com a realização de uma assistência às gestantes com qualidade, com vistas a reduzir a ocorrência de alterações, que venham a acometer a mulher e o concepto. Ademais, enfatiza-se o direito à saúde, como uma prerrogativa indispensável à apropriação da liberdade e da igualdade e a efetivação dos deveres do estado frente aos cidadãos, particularmente as mulheres que estão vivendo esse momento ímpar, que é a gravidez (SILVA; BEZERRA; TANAKA, 2012).

Diante destas informações, as ações propostas neste trabalho apoiaram-se na seguinte problemática: a atenção às gestantes ofertada pela Atenção Primária à Saúde em Aldeias Altas-MA, condiz com os parâmetros estabelecidos pelo Ministério da Saúde acerca da integralidade das ações das ações e da humanização da assistência, enfatizando os princípios do direito à saúde da mulher?

Logo, o objetivo do trabalho foi analisar a assistência ofertada às mulheres no pré-natal na Atenção Primária à Saúde, na interface com o direito à saúde.

\section{MATERIAL E MÉTODOS}

Trata-se de uma pesquisa avaliativa, descritiva e exploratória, com abordagem qualitativa, que possibilitou uma adequada relação ao objeto deste estudo, uma vez que não se constitui em um conjunto de métodos fechados, mas compreende uma abordagem flexível, abrindo espaço para que se possa ter uma compreensão e visibilidade das ações realizadas pelos enfermeiros.

O cenário da investigação foi o Município de Aldeias Altas/MA, situando-se na região leste do estado do Maranhão. E, segundo último senso, realizado em 2010, pelo Instituto Brasileiro de Geografia e Estatística (IBGE, 2016) apresenta uma população aproximada de 26.115 habitantes. Segundo dados da Coordenação da Atenção Primária à Saúde e do Sistema de Informação da Atenção Básica (SIAB), o município está estruturado com 09 Equipes de Saúde da Família (ESF), distribuídas em 03 UBS da zona urbana e 05 UBS da zona rural. Entretanto, os dados foram coletados em uma Equipe Saúde da Família do Centro de Saúde, localizada na sede da cidade, tendo como profissionais atuantes: 01médico, 01 enfermeira e 07 Agentes Comunitários de 
Saúde, além dos profissionais do Núcleo de Apoio ao Saúde da Família, fisioterapeutas, nutricionista, fonoaudiólogo e psicólogo.

Participaram da pesquisa, 10 gestantes acompanhadas pela Equipe Saúde da Família (ESF 03), tendo como critérios de inclusão na pesquisa: residirem na área de adscrição da equipe e aceitarem de livre e espontânea vontade participar da pesquisa, assinando o Termo de Consentimento Livre e Esclarecido (TCLE). Foram excluídos da investigação, as que não estivam em consonância com os itens supracitados.

As entrevistas foram realizadas pelas pesquisadoras nos dias de atendimentos na Unidade Básica de Saúde Centro de Saúde, junto a ESF 03 do município para investigar as ações do programa de pré-natal, visando colher informações dos profissionais da saúde acerca do programa pré-natal. Objetivou-se também identificar por meio das interlocuções das gestantes as ações realizadas pelos profissionais que as assistem na UBS, buscando informações sobre o desenvolvimento desse instrumento, que é indispensável para uma gravidez saudável e bem assistida.

$O$ instrumento de coleta de dados nessa pesquisa foi composto por uma entrevista semiestruturada, com a utilização de um MP3, que foram realizadas nos dias de atendimento na Unidade Básica de Saúde e versou sobre: Percepção sobre Atenção Integral à Saúde da Mulher no Prénatal, Integralidade/Humanização da Assistência à Gestantes e Ações realizadas pelos profissionais na Atenção à Mulher no pré-natal. Destaca-se que a coleta de dados aconteceu entre os meses de 01 de março a 28 de abril de 2017.

Nas respostas às perguntas abertas referentes às entrevistas com as gestantes, as falas foram submetidas à Análise de Conteúdo, proposta por Bardin (1997), que tem como propósito a compreensão do significado das falas dos sujeitos para além dos limites daquilo que é descrito. Dentre as técnicas de Análise de Conteúdo, optou-se pela Análise Temática, que busca os núcleos de sentido, os quais constituem a comunicação e cuja expressão revela algo de importante para o objeto estudado. Assim, de posse do material oriundo das entrevistas, procedeu-se a categorização, inferência, descrição e interpretação minuciosa de todo o conteúdo.

Ressalta-se que, para facilitar a compreensão das informações, os dados foram fielmente descritos e, em seguida, cada sujeito responsável pelas mesmas, foram, conforme mencionado, apresentados no texto como G1 a G10 (Gestante 1...G10), conforme ordem de aplicação dos instrumentos e para preservar suas identidades.

Outrossim, os dados foram apresentados em 3 categorias: Categoria 1: Percepção sobre Atenção à Saúde da Mulher no Pré-natal; Categoria 2: Integralidade/Humanização da Assistência à Gestante e Categoria 3: Ações realizadas pelos profissionais sobre Atenção à Mulher no pré-natal.

Os pesquisadores, comprometeram-se com as normas preconizadas pela Resolução CNS 466/12 e suas complementares, que tratam dos aspectos éticos da pesquisa envolvendo seres humanos e assegura que nenhum sujeito seja submetido à pesquisa sem seu prévio consentimento, nem sem ter garantida a sua privacidade e protegida sua integridade física e moral.

\section{RESULTADOS E DISCUSSÃO}

Os dados oriundos dos questionamentos feitos às gestantes, foram representados por meio das falas mais relevantes, de acordo com as categorias a seguir:

\section{Categoria 1: Percepção sobre Atenção à Mulher no Pré-natal}

Nesta categoria buscou-se identificar, dentre as gestantes investigadas, a percepção dessas quanto Atenção à mulher no pré-natal nos seus vários aspectos.

Os profissionais passam para nós vários exames como Ultrassom, exame de sangue, os testes de HIV e muitos outros (G3).

Não sei bem o que é Atenção Integral, mas acho que eles têm que resolver tudo (G7).

Nós recebemos todas as informações básicas na primeira consulta... isso é muito importância para o desenvolvimento da gestação (G8).

O que eu mais gosto é quando a enfermeira bota um aparelho para ouvir o coração do meu bebê (G9).

O médico e a enfermeira me dão muita atenção, me escutam bastante...eu saio mais calma quando venho me consultar (G10).

As respostas obtidas nesta primeira categoria demonstram que as gestantes possuem uma boa percepção sobre Atenção à Saúde da mulher no pré-natal, como demonstrado nas falas dos sujeitos.

Para uma Atenção à mulher no pré-natal são indispensáveis à realização de exames, acolhimento e a garantia de acesso aos serviços de saúde, em todos os níveis 
do sistema de saúde. Ressalta-se também, que a presença de profissionais preparados e com uma boa escuta, facilitam a recuperação de paciente e a redução da morbi-mortalidade entre as gestantes (PICCINI et al., 2011).

Assim, diversos aspectos influenciam a qualidade da assistência pré-natal, um dos mais importantes são os meios que os profissionais utilizam para garantir que a gestante esteja em condições seguras de manter uma gravidez, como a realização de exames laboratoriais, a ausculta dos batimentos cardo fetais, dentre outros (SOUZA; ROECKER; MARCON, 2011).

Estudos realizados por Cardoso, Mendes e VelásquezMeléndez (2012), corroboram com os desta pesquisa, ao referirem que a atuação do enfermeiro em parceria com o médico é fundamental para o desenvolvimento de atendimentos de qualidade no pré-natal.

\section{Categoria 2: Integralidade/Humanização da Assistência à Gestante}

Esta categoria apresenta resultados quanto à realização de cuidados à gestante pautados nos princípios da Política Nacional de Humanização (PNH). Dentre os quais pode se destacar o cuidado humanizado à gestante no período gestacional. As falas a seguir abordam a percepção de gestantes a este respeito.

O médico e a enfermeira me atendem muito bem, me sinto bastante acolhida (G6).

Eles sempre me chamam pelo nome $\mathrm{e}$ perguntam o que estou sentindo (G1).

Quando eu falto no posto, eles vão em minha casa, aí me consultam, me orientam (G9).

Quem me visita é só o ACS, e as vezes leva a enfermeira(G4).

Quase não recebo visitas dos profissionais do posto, só mais da Agente de Saúde (G7).

O enfermeiro dá toda assistência no prénatal, o médico fica responsável pela avaliação das intercorrências, que vão aparecendo em mim (G3).

Quando abordados sobre os diversos aspectos da Política Nacional de Humanização (PNH), a grande maioria demonstrou, através de suas respostas, um bom conhecimento sobre as diretrizes normativas dessa política. No entanto, uma análise mais minuciosa das respostas, percebeu-se que os profissionais não seguem os preceitos da PNH, uma vez que descumpre um dos seus requisitos básicos, neste caso, a visita domiciliar.

Pensar sobre a mulher e sua saúde é pensar uma nova sociedade, em que o eixo central seja a qualidade de vida do ser humano desde o seu nascimento. Dessa forma, o processo de humanização está interligado diretamente com o desenvolvimento adequado da gestação, uma vez que estudo nacional provou que gestantes atendidas em Unidades Básicas de Saúde que seguem os preceitos da Política Nacional de Humanização têm um percentil de $17 \%$ de taxa de incidência de comorbidades relacionadas à gestação, enquanto que, as Unidades que negligenciam o processo de humanização recomendado pela política de humanização, possuem uma taxa de incidência de 45\% (BRASIL, 2010).

Segundo Esposti et al. (2015), o PHPN parte do pressuposto de que a melhoria do acesso e da qualidade do acompanhamento de pré-natal é importante para a redução das altas taxas de morbimortalidade materno-infantil registradas no Brasil.

Já investigação de Esposti et al. (2015), averiguou que o SUS, apesar de orientar-se pela gratuidade do cuidado no momento de sua prestação, baseado nos princípios do cuidado universal, integral e humanizado algumas gestantes relatam grandes dificuldades para conseguir o cuidado necessário, exigindo em várias situações o uso de recursos financeiros para sua obtenção.

\section{Ações realizadas pelos profissionais sobre Atenção à Mulher no pré-natal}

Esta categoria se propõe a discutir as ações realizadas pelos profissionais de saúde, que ofertam assistência às mulheres no período pré-natal, na atenção Primária à Saúde.

De acordo com o Ministério da Saúde, as ações realizadas por profissionais no pré-natal envolvem atividades assistenciais e de educação em saúde, que são praticadas na consulta inicial e subsequentes, como solicitação de exames de rotina e complementares, que vão desde hemograma completo e Ultrassonografia, atualização em vacinação, coleta de citopatológico, mensuração e pesagem, ausculta dos batimentos cardiofetais, dentre outras. Além de atividades educativas; grupo de prevenções e promoção da saúde; visita domiciliares juntamente com o Agente Comunitários de Saúde e demais membros da Equipe de Saúde da Família (BRASIL, 2012).

Acho que é direito meu fazer os exames, 
tomar remédios, me vacinar e muitas outras atividades que tenho que realizar (G1).

Eles realizam palestras, solicitam a citologia (G2).

Me orientam pra evitar certos tipos de alimentos pra não ganhar muito peso pra não afetar o bebê, não tá tomando refrigerante, vestuário pra não usar que aperte muito a barriga e pra ter repouso (G9).

A equipe sempre faz palestras educativas, citologias oncóticas, panfletagem com os agentes de saúde comunitários na área (G4).

Eles me pedem vários exames, eu sempre procuro fazer, por que são importantes para ver se meu bebê estar bem(G6).

O médico e a enfermeira se mostram preocupados comigo, escuta o coração do meu bebê nas consultas, me pesam e me pedem a Ultrassom para ver se o coração tá batendo direitinho(G10).

De acordo com os relatos apresentados, observou-se as ações desenvolvidas pelos profissionais de saúde, que atuam na Atenção Primária à Saúde, estão em conformidade com o que é preconizado pelo Ministério da Saúde, o que permite uma aproximação maior entre profissionais e as gestantes.

A mulher preparada durante o pré-natal, por meio de informações e orientações pertinentes à gestação, passará por este período com maior segurança, harmonia e prazer, ao contrário, a falta de informação pode gerar preocupações desnecessárias e expectativas frustradas (FRANCISQUINI et al., 2010).

Vale ressaltar ainda, que a atividade educativa é essencial no processo da assistência a esta clientela. Essa atividade é de grande importância e resolutividade quando realizada de maneira adequada, pois, além de gerar autonomia da usuária, fortalece o acolhimento entre o profissional e a usuária (MARTINELLI et al, 2013).

Por direito à saúde entende-se uma questão abrangente e diversa de reclame de bens e direitos nas esferas do governo. São insumos, instalações, medicamentos, assistência em saúde, entre outros fatores a serem protegidos pelo princípio do direito à saúde. A origem da questão, ainda é duvidosa, pois existem falhas no que tange às implicações do direito à saúde no Brasil caracterizada por falhas da política pública (ROCHA, 2011).

O direito à saúde vem disciplinado no artigo 196 da Constituição Federal que dispõe que a saúde é um direito de todos, portanto, estão inseridos nessa proteção a puérpera e o neonato. Como se observa, o direito à saúde é um direito fundamental assegurado constitucionalmente pelo Estado, e de acordo com Amaral (2009), a lesão a esse direito causa o chamado danos à saúde ou biológico, que é um dano não patrimonial, em senso estrito, e moral, pelo objeto ou interesse que afeta.

No sentido de prevenir o dano à saúde da mulher e do

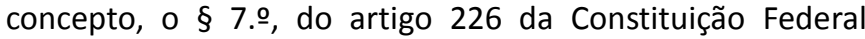
estabelece que é da competência do Estado propiciar recursos educacionais e científicos para que os genitores possam realizar o exercício do direito de implementar o planejamento familiar de forma digna e responsável. Para tanto, no sentido de regulamentar a referida competência atribuída ao Estado, foi promulgada a Lei n.o 9.263/96, denominada de Lei do Planejamento Familiar. (BRASIL, 1996), a qual prevê no inciso III, do parágrafo único do artigo 3. que as instâncias gestoras do Sistema Único de Saúde, em todos os seus níveis, obrigam-se a garantir programa de atenção integral à saúde, em todos os seus ciclos vitais, que inclua, como atividades básicas, entre outras, a assistência ao parto, ao puerpério e ao neonato.

Ademais, antes da edição da mencionada Lei, o Estado já colocava a disposição da mulher, como um direito fundamental, o atendimento pré e perinatal, através do Sistema Único de Saúde, nos termos do artigo 8.ㅇ da Lei n.으 8.069/90, conhecida como Estatuto da Criança e do Adolescente. Por certo, a assistência médica e o fornecimento de medicamentos correspondem a uma pequena parte de ações indispensáveis à efetivação do direito à saúde. Além disso, há de se desenvolver programas de medicina preventiva, de controle de doenças por meio do acesso a moradias dignas e saneamento básico, bem como de combate à desnutrição e à subnutrição (SARLET, 2005).

Os serviços de saúde possuem um custo muitas vezes alto e acabam consumindo grande parte dos recursos públicos. Assim, os recursos que já são insuficientes acabam desaparecendo quando utilizados nessa área que, com a atual tecnologia, possui tratamentos com custos bastante elevados (SCHWARTZ, 2001).

Portanto, uma vez que o direito à saúde é um direito fundamental tutelado pelo Estado, e que tal direito é garantido a todas as pessoas, quando os direitos da gestante e do nascituro são violados, em razão da falta de atendimento ou da assistência inadequada pelo Sistema Único de Saúde, porque o profissional de saúde não realizou o atendimento pré-natal, de forma qualificada, tanto a mulher quanto o 
Ministério Público tem legitimidade para exigir que a assistência seja prestada nos moldes estabelecidos pelos artigos 8. da Lei n. 8.069/90 e artigo 3.ำ parágrafo único, inciso III da Lei n.o 9.263/90. Ainda, é possível afirmar que a falta de atendimento ou a assistência inadequada e incompleta pode acarretar danos à saúde do binômio mãe/filho, e, consequentemente, a responsabilização civil do Estado.

Portanto, é notório que existem normativas importantes de acompanhamento do Pré-natal e, que de modo geral, os profissionais as conhecem, entretanto, nem sempre as ações são executadas como deveriam. Ora, porque os trabalhadores não as executam por falta de interesse próprio, em outra, porque lhes faltam os materiais e instrumentais necessários, ou ainda, porque não existe o incentivo dos gestores, tanto em termos salariais, quanto de reconhecimento profissional. Entretanto, de nenhum modo a vida e a saúde do concepto podem ser minimizadas ou desconsideradas em sua completude e dimensão.

\section{CONCLUSÕES}

Os resultados apontaram que a maioria dos profissionais de saúde, realizam as orientações às gestantes durante as consultas de pré-natal na Atenção Primária à Saúde, bem como o tratamento recebido pelas mesmas é considerado satisfatório.

Identificou-se que quanto aos aspectos da Política Nacional de Humanização, os profissionais não seguem os preceitos da $\mathrm{PNH}$, uma vez que descumpre um dos seus requisitos básicos, neste caso, a visita domiciliar.

Percebeu-se que um dos principais objetivos da Estratégia Saúde da Família (promoção de educação em saúde) está sendo realizada.

Ademais, a assistência à mulher e ao concepto é um direito garantido pelo Estado, e com o objetivo de tornar efetivo o direito à saúde, mulher, sua família e o Ministério Público tem legitimidade para exigir que o Estado cumpra sua obrigação de realizar o atendimento, o qual é fundamental e assegurado pela Constituição Federal. Assim, enfatiza-se a necessidade de intensificação das atividades de educação em saúde durante o pré-natal, em todos os aspectos, e ainda a prática de visitas domiciliares, com vistas com maior satisfação e segurança às futuras mães.

\section{REFERÊNCIAS}

ALMEIDA, A. D.; BARROS, G. D.F. Cuidados de enfermagem na transição do papel materno entre puérperas. Rev. Eletr. Enferm. Goiânia/GO, v. 9, n. 2, p. 457- 475, jul/ago, 2013.
ANDREUCCI, C. B.; CECATTI, J. G. Desempenho de indicadores de processo do Programa de Humanização do Pré-Natal e Nascimento no Brasil: uma revisão sistemática. Caderno Saúde Pública, Rio de Janeiro, v. 27, n. 6, p. 1053-1064, junho, 2011.

BARDIN, L. Análise de conteúdo. São Paulo: Ed. Vezes, 1997.

BRASIL. Ministério da Saúde. Assistência ao Planejamento Familiar. Brasília, Centro de Documentação do Ministério da Saúde, 2006.

Ministério da Saúde. Secretaria de Atenção à Saúde. Departamento de Atenção Básica. Manual do prénatal de baixo risco. Brasília: Ministério da Saúde, 2010.

CAHALI, Y. S. Dano moral. 3. ed. rev., ampl. e atual. conforme o Código Civil de 2002. São Paulo: Revista dos Tribunais, 2005.

COFEN. Conselho Federal de Enfermagem. Projeto Lei 2295/2000. Brasília, DF: Ministério da Saúde; 2004.

CORRÊA, C. R. H, BONADIO, I. C, TSUNECHIRO, M. A. Normative prenatal evaluation at a philanthropic maternity hospital in São Paulo. Rev Esc. Enferm USP, São Paulo, v. 45, n. 6, p. 1293-1300, junho, 2011.

DRUMOND, E. F.; MACHADO, C. J.; FRANÇA, C. J. Óbitos neonatais precoces: análise de causas múltiplas de morte pelo método Grade of Membership. Cad. Saú. Púb. Rio de Janeiro/RJ, v. 27, n. 1, p. 67-77, jan. 2011.

ESPOSTI, C. D. D. et al. Representações sociais sobre o acesso e o cuidado pré-natal no Sistema Único de Saúde da Região Metropolitana da Grande Vitória, Espírito Santo. Saúde Soc. São Paulo, São Paulo, v. 24, n. 3, p.765-779, mar., 2015.

FRANCO, J. et al. Percepção da equipe de enfermagem sobre fatores causais de erros na administração de medicamentos. Rev Bras Enfermagem, Brasília-DF, v. 63, n. 6, p. 927-932, nov/dez, 2010.

MARTINS, A. F; DE PAULA, P. A. Evaluation of process indicators program for humanization of prenatal and birth. Revista de Enfermagem da UFPI, Teresina, v. 3, n. 1, p. 3945, jan./mar., 2014.

MOIMAZ, S. A. S. et al. Sistema de Informação Pré-Natal: Sistema de Informação Pré-Natal: Sistema de Informação Pré-Natal: análise crítica de registro análise crítica de registros em um município paulista os em um município paulista. Rev Bras Enferm, Brasília, v. 63, n. 3, p. 385-390, maio-jun., 2010.

ORTEGA, M. C. B. et al. Formação acadêmica do profissional de enfermagem e sua adequação às atividades de trabalho. Rev. Latino-Am. Enfermagem, São Paulo/SP, v. 23, n. 3, p. 404-410, maio-jun, 2015.

PICCINI, E. T. O et al. Características da utilização de serviços de atenção básica à saúde nas regiões Sul e Nordeste do Brasil: diferenças por modelo de atenção. Ciênc. Saú. Col., 
Rio de Janeiro/RJ, v. 16, n. 11, p. 98-110, nov, 2011.

POLIDO, C. G. Vivências maternas associadas ao aleitamento materno exclusivo mais duradouro: um estudo etnográfico. Acta paulista de enfermagem, São Paulo, v. 24, n. 5, p. 624-30, fevereiro, 2010.

ROBAZZI, M. et al. Alterações na saúde decorrentes do excesso de trabalho entre trabalhadores da área de saúde. Rev Enferm UERJ, Rio de Janeiro, v. 20, n. 4, p. 526-532, 2012.

SILVA, B. F.; BEZERRA, M. C.; TANAKA, A. C. A. Mortalidade neonatal precoce em Caxias do Sul: um estudo de coorte. Jor. Pediatr, Rio Janeiro/RJ, v. 86, n. 06, p. 200-206, nov/dez, 2012.

SILVA, B. et al. Jornada de trabalho: fator que interfere na qualidade da assistência de enfermagem. Texto Contexto Enferm, v. 15, n. 3, p. 442-448, 2006.

SOUZA, V. B.; ROECKER, S.; MARCON, S. S. Ações educativas durante a assistência pré-natal: percepção de gestantes atendidas na rede básica de Maringá-PR. Rev Eletr Enferm., Belo Horizonte/MG, v. 13, n. 2, p. 199-210, jun., 2011.

VALENTE, M. M. P. Q et al. Assistência Pré-natal: um olhar sobre a qualidade. Rev. Rene. Fortaleza, v. 14, n. 2, p-6358, abril, 2013.

XIMENES NETO, R. T. et al. Re-significando a dor e superando a solidão: experiências do parto entre adolescentes de classes populares atendidas em uma maternidade pública de Salvador, Bahia, Brasil. Cad. Saú. Púb, Rio de Janeiro/RJ, v. 42, n. 1, p. 456-463, jan., 2008.

Submissão: 05/01/2019

Aprovado para publicação: 16/07/2019 